\title{
Quality of life of the elderly
}

\section{KEYWORDS}

quality of life, healthy aging, elderly people

\begin{abstract}
Rosenfeld Lilia, Quality of life of the elderly. Kultura Społeczeństwo - Edukacja nr 2(14) 2018, Poznań 2018, pp. 297-305, Adam Mickiewicz University Press. ISSN 2300-0422. DOI 10.14746/kse.2018.14.20.

Drawing on Simone de Beauvoir's call for a change perspective on old age, as perceive, this article reviews the literature for factors and aspects that influence the quality of life of the elderly. Simone de Beauvoir saw old age as the individual's unending struggle with the indifference that elderly people encounter, and with pain and illnesses. The improvement in the situation of the elderly cannot be accomplished by merely raising pensions and providing fair housing; rather it is necessary to have a more profound change - a change of life (de Beauvior, 1996). Hence, improving the quality life of elderly people necessitates a more holistic approach, that considers physical functioning, mental, social, environmental and cognitive condition in addition to traditional aspects involving health and economic aspects.
\end{abstract}

Simone de Beauvoir in her work "The Coming of Age" saw the period of old age in the person's life as half-life and half death. She described a reality in which most people who reach this age, accept it with great sadness or rebellion and find it difficult to bear. Old age is the individual's unending struggle with the indifference that the old person encounters, with the pain he bears, with the illnesses that accompany him, with the loss of wisdom (de Beauvoir, 1996).

Godwin et al. (2015) in his research on the group of elderly in Canada conveys criticism about the reference of the rest of society towards the elderly as a unit. Advanced age in itself does not harm the individual's perception of qual- 
ity of life. Aging is a long but natural process that causes the individual's values and norms not only to obtain different weight, but also to change completely. Following significant life events, people change the priorities of their values, and consequently their perception of mental wellbeing and quality of life. One of the most significant principle for elderly people is the lack of dependence on others and maintaining independence in everyday activity. A value which is not placed in a high priority at a younger age, as functional ability at a young age is taken for granted (Godwin, et al., 2015). However, values are not universal, but rather dependent on the culture in which the social interaction exists. Another significant consideration for quality of life in old age is the quality of death. Like the quality of life, the quality of death is a subjective concept, depending on the individual's culture, set of values, and health, in case of a chronic sickness, its severity, and its influence on the cognitive and physical functioning in everyday life (Forte, Boreham, De Vito, Pesce, 2015).

The psychological resources and abilities of the individual are especially noteworthy, including self-efficacy, self-achievement, and ego-integrity. These factors are held essential to the life of every individual, regardless of age. They remain vital to the achievement and preservation of the quality of life on a high level at old age. Another factor that influences the perception of quality of life is the presence of leisure activities in life. Leisure activities constitute strong prognostic indicators of good aging. In general, the lack or low level of leisure activities in life, predicts the appearance of depression in adult life. Depression detrimentally influences wellbeing and constitutes a very significant risk factor for health at all ages, especially in old age (Han et al., 2015).

Similarly, according to Erikson's theory, throughout the course of life, from childhood to death, the individual is engaged in the resolution of the conflicts between internal biological needs and social requirements. Erikson divided the course of life into eight stages, with each stage being characterized by a task or a conflict that demands a solution. The person's development depends on providing solutions to conflicts in the stages of life (Erikson \& Joan, 1997). The successful or unsuccessful handling of those conflicts instills different tools, and with them develop a good ability or the lack of such ability to cope with the last stage of life 'old age'. Erikson believed that from the age of fifty the retrospective look at life influences the person's negative or positive perception of his old age. At this stage, the elderly person summarizes the achievements and failures in his life. When a person evaluates that he has more achievements in life than failures, then he will feel 'ego integrity'. In other words, s/he accepts his life, accepts his feelings and the events that occurred, he feels satisfied with his old age, and he accepts death. 
However, when the person feels despair and fear of death, he displays a sense of bitterness, regret for what has been accomplished in life, and a feeling that he has missed out. This person will tend to depend on others and will experience great difficulty accepting the end of life.

When coping with the conflict between despair and ego integrity is successful, wisdom is achieved and maintaining independency in decision making is more likely. Wisdom enables elderly people to profoundly understand life and accept it without negative emotions, such as frustration and a feeling of having missed opportunities. The achievement of ego integrity in old age not only brings with it positive emotions such as acceptance of life events and death, but also leads to better health. However, a failure to solve the conflict between despair and ego integrity may lead the individual to give up the responsibility for making independent decisions, even day to day ones. In parallel, the individual may become emotionally closed, with declining levels of physical activity, and diminished social and family relationships.

The same is true for health. When elderly people have acquired wisdom in old age, a higher level of self-management is identified than with elderly people who are despaired and frustrated. Moreover, although elderly people suffer from a decline in their physical and cognitive abilities, those who achieved wisdom show a better level of health than those who feel disappointment and despair regarding events in their lives. In addition, elderly who independently take the decision to move to an institution tend to live longer. Elderly who have successfully acquired tools in the early stages of life have better chances to adjust to their changing social role and accept the decline in the physical ability (Perry, Hassevoort, Ruggiano, \& Shtompel, 2015). Results of the research study performed among elderly people in Korea indicate that taking medications for balanced chronic illnesses is not at all related, in the opinion of the elderly, to quality of life and therefore does not detrimentally influence the perception of quality life, although unbalanced severe or chronic illness detrimentally influences the quality of life, as perceived by the elderly (Han et al., 2015).

Furthermore, elderly who have reached ego integrity are better in identifying opportunities in life and in using them for own benefit and for the benefit of the surrounding environment. In other words, they are socially active, contribute to society and cope better with life crises. In contrast, elderly who were unsuccessful in solving the conflict in the stage of old age experiences a decline in all areas of life. Thus, those elderly people, alongside experiencing cognitive and physical deterioration, surrender autonomy in making decisions and tends to develop greater and worse chronic illness than elderly who have resolved the conflict more suc- 
cessfully in the stage of old age (Erikson \& Joan, 1997; Perry, Hassevoort, Ruggiano, \& Shtompel, 2015).

According to de Beauvoir (1996), in old age people suffer from the lack of inventiveness or their ability to invent is hampered by society. In her opinion, engaging aging people, with the help of their relatives, in social activities, politics and creative activities may provide a solution. De Beauvoir writes that life has value in old age when there is a relationship with others, when there is love and friendship.

Additional factors that contribute to the explanation of the perception of the quality of life are consistent reciprocal relations, sense of social belonging, and feeling of love and concern of others. These are main components in the achievement of quality of life, both in sickness and in health. The presence of chronic mental or physical illness is far less harmful to the perception of the quality of life than the lack of happiness for long periods (Connell, O'Cathain, \& Brazier, 2014). Also, regular reciprocal relations with the neighborhood improve the elderly person's control and contribute to his autonomy, enjoyment, and self-fulfillment in aging (Mottus, Gale, Starr, Deary, 2012). Social belonging, feeling of love and concern of close people, as well as caring for people even in sickness are components found at the basis of the perception of quality of life of all those who participate in the process (Ducharme, Geldmacher, 2011).

According to Guan et al. (2015) residing in a familiar environment becomes more crucial as one grows older. Elderly people prefer to remain with their adult children in a familiar environment where they have lived before. In contrast to cultural values, the reality dictates that children leave their parents' home and their familiar environment, in search of a better life. As a result, elderly people are faced with the dilemma either to shift their residence to an unfamiliar environment or remain alone. The lack of fit between the elderly person's ability and desire to choose own future residence leads to a gap between reality and own preferences. This gap harms the sense of self-efficacy in decision-making, as well as the sense of life satisfaction.

The lack of a comfortable environment or reciprocal relations with society harms the elderly person's physical and mental health and reduces his assessment of the level of quality of life. Likewise, the presence of neighbors constitutes a factor that protects against cardiovascular disease, and ensures the low levels of pain related to daily activities, shortens periods of recuperation from severe illnesses, and improves the elderly person's general health. Social isolation increases the risk of depression and anxiety. In contrast, being with neighbors, in a good, supportive, and appropriate environment, constitutes protective factors and prevents the 
appearance of deterioration in the elderly person's emotional and mental situation (Mottus, Gale, Starr, \& Deary, 2012).

In old age, as in youth, the quality of life with reference to the loss of physical abilities is influenced by the individual life style throughout the entire life. The goal of a healthy, positive, and active aging raises the requirement for understanding the special needs of old age. Healthy aging is a long process, which entails, among other, the promotion and maintenance of the physical, mental and social wellbeing (Han et al., 2015). Simone de Beauvoir (1996) in her work recommends planning for old age. In her opinion, such planning includes not only the saving of money and the choice of residence upon retirement but also the acquisition of different hobbies and living a life of involvement that will provide an opportunity for the continuation of a real life after retirement.

Despite prejudices in society regarding elderly people, who are perceived as a group of people with a poor functional level, there are many elderly people in a good health condition. They live alone without any outside help and their level of social activities is high. They contribute to the community in which they live and are still involved in the social life of their families and friends. Therefore, age is not the only factor influencing the quality of life (Godwin et al., 2015). When the relation between the quality of life and the functional ability of the individual is discussed, it is important to take into consideration not only the level coping with everyday physical activities but also the level of cognitive abilities in the performance of different tasks. There is a significant relation between quality of life and everyday physical functioning. little is known today about the correlation between the cognitive level of functioning and the perception of quality of life. Research has identified a correlation between quality of life in old age and cognitive abilities in youth. However, few works have attempted to prove and explain the correlation between cognitive abilities in old age and quality of life (Forte, Boreham, De Vito, Pesce, 2015). Typically, when quality of life and mental wellbeing are examined, physical disabilities and physical illness are taken into consideration. However, cognitive abilities are neglected, as well as mental illnesses (such as schizophrenia, personal disorders, anxieties) (Connell, O’Cathain, Brazier, 2014).

The process of aging is for the most part influenced by other factors, such as education, family status, and health situation. It is customary to assume that health condition worsens as people grow older. However, the fact that not every old person is helpless and isolated should be kept in mind. Furthermore, elderly people with balanced chronic illnesses may not necessarily need help in the performance of everyday activities (Godwin et al., 2015). Nevertheless, researchers agree that in advanced adulthood the individual's level of satisfaction with life depends on 
good physical functioning in everyday activity. Though some studies maintain that a good physical level in old age reflects a higher fit to good health condition, the reverse is true. When there is a decline in everyday functioning, a decline in health condition can be expected. Furthermore, researchers maintain that excellent physical functioning greatly contributes to the individual's quality of life, mental wellbeing, and satisfaction with life (Forte, Boreham, De Vito, Presce, 2015).

Good health in old age contains lack of depression, high self-efficacy, coping with loneliness, and avoidance of social isolation. When all these needs are fulfilled in a satisfactory manner, even in the presence of certain chronic illness, the self-evaluation of the quality of life is at a high level. Isolation and social loneliness are the factors that bear a negative influence on people's wellbeing, and this influence becomes more decisive in old age (Han et al., 2015).

A decline in social activities and in the number of social relations, loss of close people, reduction in reciprocal relations, change of residence following a decline in the physical ability or health deterioration may harm elderly people's mental wellbeing (Han et al., 2015). Forte, Boreham, De Vito, and Pesce (2015) maintain that the promotion and maintenance of the mental wellbeing while aging are possible through the performance of moderate physical activity and healthy life style. Walking in old age, physical activity constitutes a protective factor against cognitive and functional deterioration (Forte, Boreham, De Vito, \& Pesce, 2015). Conversely, a study by Han et al. (2015) indicates that mental wellbeing and mortality among aging people are significantly influenced by their beliefs and perception of their health as 'good' or 'poor', regardless of the objective severity of the illness in medical terms. Spiritual and cultural beliefs have a substantial influence on the perception of the family quality of life; they reinforce emotional support in the family and help maintain reciprocal relations with the community. The importance of beliefs and the strength of their influence on the perception of quality of life rise with age. In other words, the process of aging is accompanied by the strengthening in the beliefs (Bertelli, Biaco, Rossi, Scuticchio, Brown, 2011).

The rise in the life span in the population, which characterizes most of the Western countries, requires decision makers to understand how to maintain health at a good level along with high level of quality of life in the later adulthood and old age (Forte, Boreham, De Vito, Pesce, 2015; Mottus, Gale, Starr, Deary, 2012). General aging of the population of the world has become a main topic discussed in many forums - public, health, and social. In many countries, the burden on the health systems caused by the aging of the population is constantly increasing. Elderly people are reaching ages in which their overall function is diminished and the risk of chronic disease increases. A decline in the physical abilities and person- 
al illness requires different resources. Moreover, the burden of the care of the sick or healthy elderly person relies on the economic capacity of the family in which the person ages. Frequently children of elderly parents are expected to take the burden of the elderly person's care upon themselves. For example, in Taiwan, which is influenced by the principles of the Buddhist religion, it is customary for children to play an active role in the care of their aging parents, since the parents had cared for them in childhood. However, modernization has detrimentally influenced the ability of the family to provide a security net for the aging person, while searching for a better economic life. Children of elderly parents move their residence to new places and leave their aging parents behind. Today it is clear that, the family as a social unit can no longer bear the burden of care of elders alone, without help from society and state (Manasatchakun, Chotiga, Roxberg, Asp, 2016). In China, as in many countries of Asia, the son bears the responsibility for the care of his parents at a late age. However, following the rapid aging of the population and the policy of family planning and birthrate reduction in China there is a considerable decrease in the ability of the family to care for the elderly within the family. Therefore, in recent years, the percentage of the elderly who live outside of their families is steadily increasing. Today, adults in China, in contrast to tradition and culture, live alone, with their spouse, or in retirement homes (Guan, Li, Sun, Wang, \& Wu, 2015).

The process of aging, regardless of the medical condition, requires high economic investment, not only on the personal-family level but also on the level of society and the State. To properly evaluate the needs of the elderly and to enable an effective intervention, it is necessary to research the perception of people regarding the quality of life from childhood to old age. Today, there is insufficient knowledge regarding the perception of the quality of life among the elderly. In addition, there is confusion regarding the different concepts in the literature. Some remain undefined or are defined differently among the researchers. For instance, healthy old age is defined as devoid of illnesses or as active old age or functioning without help, and so on (Godwin et al., 2015; Manasatchakun, Chotiga, Roxberg, Asp, 2016).

Godwin et al. (2015) maintain that the group of elderly people is one of the most heterogeneous groups in society. The division that exists today into healthy or ill is very sparse and does not reflect the true difference. Their study among elderly people, conducted in Canada, indicates that there is a group of elderly above the age of 80 who function independently in everyday activities, are cognitively alert, involved in the society, community, and family, are highly educated, and with a high motivation to integrate socially. The needs and desires of these elderly are 
different from the requirements of the elderly with different backgrounds. Shaw et al. (2016) claim that the implementation of a humanistic approach on the social level towards the elderly person in society will help unite all relevant issues and build a holistic approach that views the elderly from their perspective and not merely as a burden on the health services. Healthy aging is not only the characterization of the elderly person's needs; rather, it is a process of decisions that the individual makes in many areas in his life for the purpose of promoting and preserving own mental spiritual wellbeing and physical health. All these constitute a significant part in the influence on the elderly population's evaluation and perception of the quality of their lives (Han et al., 2015).

Simone de Beauvoir maintained that society has fated the elderly to misery and to poverty, to inappropriate residence, and to loneliness. She sees this type of attitude towards the elderly to be the complete responsibility of society, and blames contemporary society for the creation handicap and misery of old age. De Beauvoir holds that society is filled with painful obstacles and has a poor attitude in moral terms. In reality, the elderly people are powerless and empty-handed, and when their power leaves them they are 'thrown away' by society, tossed to the margins as trash. The elderly person is weak and all that remains to him from all he had in life are his two eyes - to cry. In her opinion, this must not happen. De Beauvoir maintains that improvement in the situation of the elderly cannot be accomplished only by raising the pensions, providing fair housing, organizing leisure activities; rather it is necessary to have a more extreme and profound change - a change of life (de Beauvoir, 1996).

\section{References}

Beauvior S. de (1996). The coming of Age. (P. O’brain, Trans.) New York.

Bertelli M., Bianco A., Rossi M., Scuticchio D., Brown I. (2011). Relationship between individual quality of life and family quality of life for people whith intellectual disability living in Italy. "Journal of Intellectual Disability Research” 55 (12), pp. 1136-1150.

Connell J., O'Cathain A., Brazier J. (2014). Measuring quality of life in mental health: Are we asking the right questions? "Social Science \& Medicine" 120, pp. 12-20.

Ducharme J.K., Geldmacher D.S. (2011). Family quality of life in dementia: A qualitative approach to family - identified care priorities. "Quality of Life Research: An International Journal of Quality of Life Aspects of Treatment, Care and Rehabilitation" 20, pp. 1331-1335.

Erikson E., Joan M. (1997). The life cycle completed: extended version. New York.

Forte R., Boreham C.A., De Vito G., Pesce C. (2015). Health and quality of life perception in older adults: The joint role of cognitive efficiency and functional mobility. "Environmental Research and Public Health" 12, pp. 11328-11344. 
Godwin M., Pike A., McCrate F., Parsons K., Parsons W., Pitcher H., Sclater A. (2015). The healthy aged. "Canadian Family Psysician", pp. e142-e147.

Guan J., Li H., Sun H., Wang T., Wu W. (2015). The impact of a discrepancy between actual and preferred living arrangements on life satisfaction among the elderly in China. "Clinics" 70 (9), pp. 623-627.

Han K., Lee Y., Gu J., Oh H., Han J., Kim K. (2015). Psychosocial factors for influencing healthy aging in adults in Korea. "Health and Quality of life Outcomes" 13, 31, pp. 1-10.

Manasatchakun P., Chotiga P., Roxberg A., Asp M. (2016). Healthy ageing in Isan - Thai culture a phenomenographic study based on older persons' lived experiences. "International Journal of Qualitative studies on Health and Well-being” 11, pp. 1-8.

Mottus R., Gale C.R., Starr J.M., Deary I.J. (2012). “One the street where you live”: Neighbourhood deprivation and quality of life among community - dwelling older people in Edinburgh, Scotland. "Social Science \& Medicine" 74, pp. 1368-1374.

Perry T.E., Hassevoort L., Ruggiano N., Shtompel N. (2015). Applying Erikson's wisdom to self-management practices of older adults: Findings from two field studies. "Research Aging" 37(3), pp. 253-274. 